\title{
Intensifier la culture du maïs pour améliorer la sécurité alimentaire : le producteur du Nord Cameroun y a-t-il intérêt ?
}

\author{
Hervé Guibert $^{1, *}$, Pinardel Kenne Kueteyim ${ }^{2}$, Jean-Paul Olina Bassala ${ }^{3}$ et Mathurin M'Biandoun ${ }^{4}$ \\ ${ }^{1}$ CIRAD, UPR AÏDA, 34398 Montpellier, France \\ 2 IRAD, LASPEE, Nkolbisson, Yaoundé, Cameroun \\ 3 IRAD, Centre Régional de Maroua, Maroua, Cameroun \\ 4 IRAD, Station de Bocklé, Garoua, Cameroun
}

\begin{abstract}
Résumé - L'intensification de la culture du maïs, majoritairement destinée à l'autoconsommation rurale et à l'approvisionnement des villes, est une opportunité pour améliorer la sécurité alimentaire des régions septentrionales du Cameroun et limiter la pression foncière. Pour étudier sa faisabilité, trois niveaux d'intensification ont été testés en 2013 dans 32 champs de maïs à proximité de Garoua dans la région du Nord, 16 considérés comme fertiles par les producteurs et 16 comme dégradés : (i) la conduite habituelle par le producteur (CP), (ii) un premier niveau d'intensification (NI1) correspondant aux recommandations actuelles de la vulgarisation agricole et (iii) un second niveau d'intensification (NI2) avec, par rapport à NI1, un renforcement des amendements, un traitement de semences amélioré, de meilleures densité et contrôle des mauvaises herbes. CP et NI1 ont des rendements proches dans les deux villages : en moyenne 2,3 et 1,3 tha $^{-1}$, respectivement sur parcelles fertiles et dégradées, indiquant que les producteurs suivent assez bien les recommandations de la vulgarisation agricole. Les rendements des parcelles sous NI2 sont significativement supérieurs aux autres, en moyenne de $1 \mathrm{t}^{-1} \mathrm{a}^{-1}$ sur le site de Ngong où la pluviosité est la plus faible et $1,5 \mathrm{t} \mathrm{ha}^{-1}$ sur le site de Gashiga. Curieusement, le gain de rendement apporté par NI2 est identique sur parcelles fertiles comme dégradées, indiquant que la dégradation de ces dernières, avérée par leur moindre production, permet néanmoins une réponse à une intensification accrue. Malgré ces résultats, la profitabilité d'une intensification accrue au prix actuel du maïs, notamment celui ayant cours au moment de la récolte, n'est pas établie. Un meilleur prix rendrait profitable l'intensification et la production accrues de la culture du maïs dans ces régions, comme le pratiquent déjà en partie certaines exploitations dites émergentes, qui maitrisent mieux la commercialisation de leurs productions.
\end{abstract}

Mots clés : maïs / intensification / sécurité alimentaire / Afrique sub-saharienne / analyse économique

\begin{abstract}
Intensification of maize cropping systems to improve food security: is there any benefit for Northern Cameroon farmers? The intensification of maize cultivation, mainly for rural consumption and cities supply, is an opportunity to improve food security in the Northern regions of Cameroon, and to face land constraints. To study its feasibility, three levels of intensification have been tested in 2013 in 32 maize fields near Garoua in the Northen Region, of which 16 considered fertile by farmers, and 16 as degraded: (i) the conduct by the producer (CP), (ii) a first level of intensification (NI1) corresponding to the currently recommended agricultural specifications, and (iii) a second level of intensification (NI2), which compared to NI1 benefited of a strengthening in fertilization, improved seed treatment, better density and weeds control. CP and NI1 have close yields in both villages: an average of 2.3 and $1.3 \mathrm{t}$. $\mathrm{ha}^{-1}$, respectively on fertile and degraded fields, proving that producers follow pretty much the broadcast technical specifications. Yields on plots under NI2 are significantly superior to others, on average of $1 \mathrm{t}$. $\mathrm{ha}^{-1}$ at the Ngong site where rainfall is lower, and $1.5 \mathrm{t}^{-h a^{-1}}$ at the Gashiga site. Curiously, the yield gain provided by NI2 is identical on fertile plots as on degraded ones, indicating that the degradation of the recent years, demonstrated by their lower production, nevertheless allows a response to increased intensification. Despite these results, the profitability of an increased intensification at the current price of corn, especially the one at the harvest time, is not established. A better price would allow
\end{abstract}

\footnotetext{
* Auteur de correspondance : herve.guibert@cirad.fr
} 
the profitability of an increased intensification and production of corn in these areas as already partially practiced by some emerging farms, as they are named, which have more control over the marketing of their products.

Keywords: maize / intensification / food security / Sub-saharan Africa / economic analysis

\section{Introduction}

$\mathrm{Au}$ Cameroun, la région administrative du Nord a produit en 2010, selon le ministère de l'Agriculture et du Développement rural (Minader, 2012), un peu moins de 211000 tonnes de maïs. Cette production est essentiellement autoconsommée $(70 \%)$ et sert à l'approvisionnement des villes, principalement celles des trois régions du nord du Cameroun (Nzossié et al., 2010). Parallèlement, le service des Nations unies (UN-OCHA, 2016) indique qu'environ un ménage sur cinq se trouve en situation d'insécurité alimentaire dans les régions de l'Extrême-Nord, du Nord, de l'Adamaoua et de l'Est du Cameroun. Le niveau de production du maïs et sa stabilité sont essentiels pour assurer la sécurité alimentaire des populations de ces régions. Les rendements obtenus par les exploitations familiales de la région Nord ont été estimés par plusieurs études. Ainsi, des diagnostics réalisés sur deux villages de référence de cette région indiquent des rendements moyens, en 1999 sur 439 exploitations, de 1805 kg.ha ${ }^{-1}$ (Havard et al., 2000 ) et en 2000 sur 479 exploitations de $2030 \mathrm{~kg} \cdot \mathrm{ha}^{-1}$ (Havard et Abakar, 2001). Naudin et al. (2010) sur 22 parcelles de maïs conduites par les producteurs et selon leurs pratiques habituelles entre 2004 et 2006 dans cette région, observent des rendements moyens de $1940 \mathrm{~kg} \cdot \mathrm{ha}^{-1}$. En 2010, le ministère de l'Agriculture et du Développement rural (2012) annonce un rendement régional moyen de $1970 \mathrm{~kg} \cdot \mathrm{ha}^{-1}$. Ces différentes données indiquent un niveau de rendement observé en dessous du potentiel, avec de fortes fluctuations d'une année sur l'autre et en stagnation depuis une dizaine d'années. En essais en station, des rendements en maïs-grain entre 4 et 5 t.ha $^{-1}$ sont régulièrement obtenus en zone soudanienne (Menyonga et al., 1994). Quelques années après l'expansion de la culture du maïs en Afrique de l'Ouest, Fusillier (1995) notait déjà chez les producteurs des rendements entre 1,5 et 2,5 t.ha $^{-1}$. Des progrès sont-ils encore possibles ? Un renforcement de l'intensification permettrait-il d'améliorer la sécurité alimentaire des populations locales ? Les niveaux d'intensification appliqués par les producteurs dépendent étroitement des prix auxquels ils peuvent vendre leurs produits. Au niveau international, l'indice FAO des prix des céréales sur le marché mondial avait atteint la valeur de 232 (base 100 en 2003) durant la crise alimentaire de 2008 ; il a progressé jusqu'à 241 en 2011 et fluctue depuis autour de 150. Sur le long terme, l'évolution des prix internationaux semble orientée à la hausse, malgré la baisse de ces dernières années. Au niveau régional africain, la volatilité des prix régionaux ne reflète pas intégralement celle des prix internationaux (Minot, 2014). Les nouvelles contributions demandées à l'agriculture (nourrir une population mondiale en forte croissance, fournir des substituts aux énergies fossiles) tout en réduisant ses impacts négatifs sur l'environnement, pourraient induire une évolution du prix du maïs à la hausse comme l'estime l'OCDE/FAO (2008). La hausse du prix du maïs permettrait d'envisager une augmentation des coûts de production nécessaire à une intensification de la culture.
Dans ce contexte, notre étude a pour objectif d'évaluer la profitabilité d'une intensification accrue de la culture du maïs dans la région du Nord au Cameroun et d'en déterminer les contraintes, en particulier celle du niveau de fertilité des sols. L'intensification proposée concerne l'ensemble de l'itinéraire technique, et pas seulement l'apport de fertilisants, afin d'éviter qu'un état cultural défavorable (mauvaise densité de culture ou enherbement excessif par exemple) ne remette en cause le résultat. En effet, l'amélioration d'une seule opération culturale, le reste de l'itinéraire étant inchangé, ne permet pas d'accroître significativement le rendement (Sissoko et al., 2015). L'étude vise également à évaluer la rentabilité d'un niveau plus élevé d'intensification en fonction du prix d'achat du maïs au producteur, car l'intérêt d'une nouvelle technique de production peut se révéler peu pertinente dans le contexte économique actuel, mais le devenir en cas d'une évolution favorable du prix au producteur.

\section{Matériels et méthodes}

\subsection{Les sites d'étude}

L'étude a été menée chez des producteurs de deux sites de la région du Nord au Cameroun pendant la saison de culture 2013: (i) le site de Ngong avec les villages de Normandi et Kawtal au sud de Garoua ( $\left.09^{\circ} 03^{\prime} \mathrm{N}, 13^{\circ} 27^{\prime} \mathrm{E}\right)$ et (ii) le site de Gashiga avec le village de Ngabala au nord de Garoua $\left(09^{\circ} 34^{\prime}\right.$ $\left.\mathrm{N}, 13^{\circ} 21^{\prime} \mathrm{E}\right)$.

Brabant et Gavaud (1985) ont référencé les sols des deux sites comme des sols ferrugineux différenciés sur grès quartzeux. Ce type de sol constitue une part importante des sols cultivés du Bassin de la Bénoué.

Le cumul de pluviosité annuelle a été respectivement de 691 et $800 \mathrm{~mm}$ sur les sites de Ngong et Gashiga. Ils sont faibles et contrastés, la répartition ayant été cependant correcte (Fig. 1 et 2). À Ngong, le cumul annuel observé en 2013 a été le troisième plus bas de la période 1965 à 2013, dont la moyenne est de $922 \mathrm{~mm}$. À Pitoa, qui se trouve à proximité de Gashiga, la moyenne des cumuls annuels de pluviosité était de $918 \mathrm{~mm}$ entre 1965 et 2013. La pluviosité de 2013 a été déficitaire à Gashiga, mais dans une moindre mesure par rapport à Ngong.

\subsection{Le plan expérimental et les modalités comparées}

Dans chacun des deux sites, les producteurs ont été invités à classer, selon leur perception et indicateurs habituels, leurs champs en deux catégories : «dégradé » ou «fertile ». Pour réaliser ce classement, les producteurs se sont référés aux productions obtenues sur leurs champs dans le passé avec l'itinéraire technique qu'ils ont l'habitude de pratiquer. M'Biandoun et al. (2006) ont montré que la connaissance du niveau de fertilité des parcelles par les producteurs est conforme aux analyses que peuvent en faire les scientifiques. 


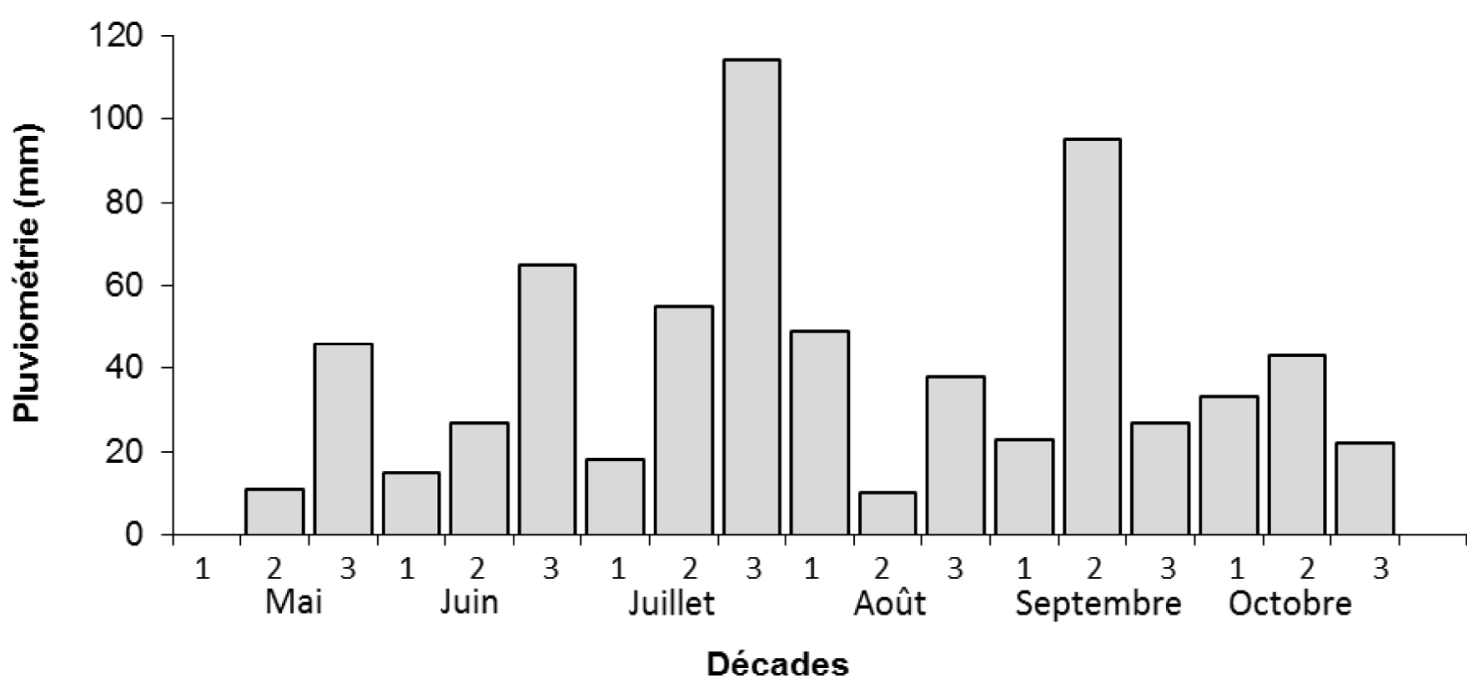

Fig. 1. Pluviosité décadaire sur le site de Ngong, Nord Cameroun, campagne 2013.

Fig. 1. Decadal rainfall on the site of Ngong, Northern Cameroon, crop year 2013.

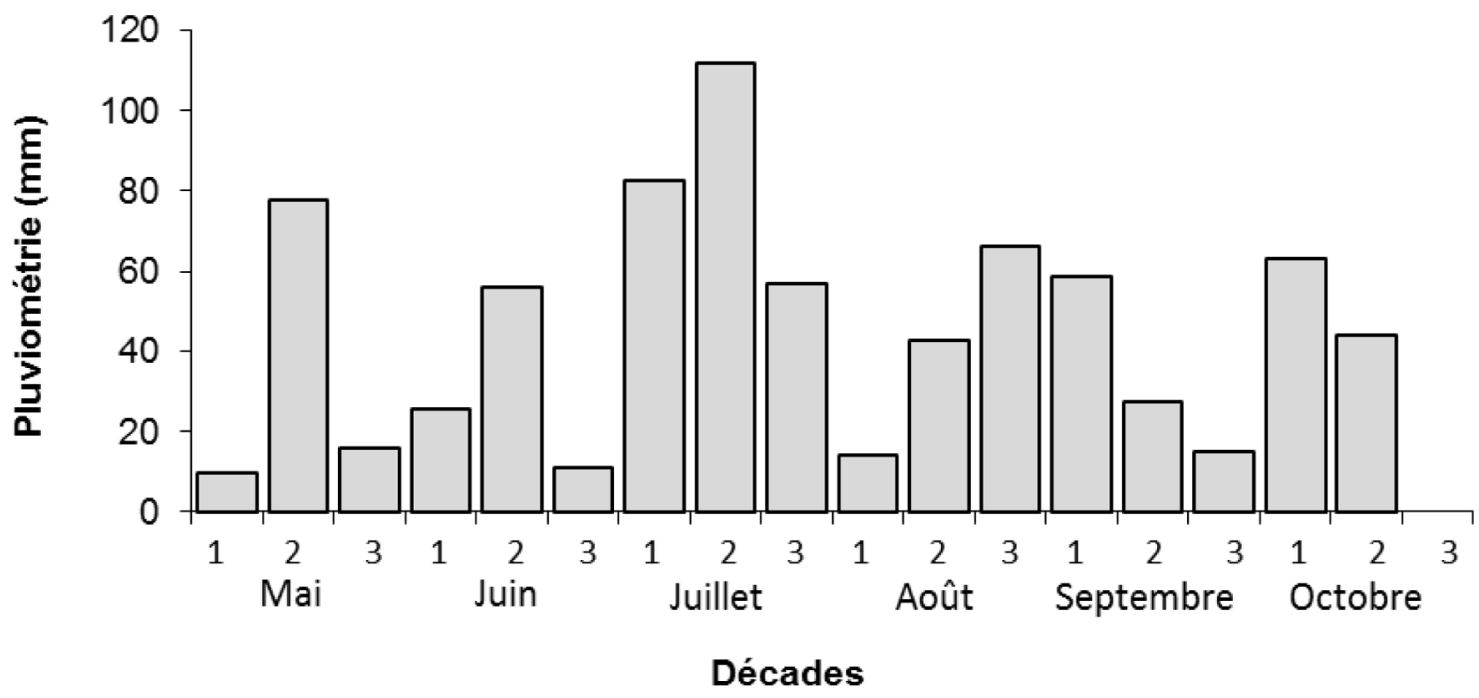

Fig. 2. Pluviométrie décadaire sur le site de Gashiga, Nord Cameroun, campagne 2013.

Fig. 2. Decadal rainfall on the site of Gashiga, Northern Cameroon, crop year 2013.

Dans chaque champ, un essai en blocs de Fischer à un facteur (niveaux d'intensification) a été installé avec trois modalités et deux répétitions. Les trois modalités étaient : (i) conduite $\mathrm{du}$ producteur $(\mathrm{CP})$, (ii) premier niveau d'intensification (NI1) et (iii) second niveau d'intensification (NI2). Chaque parcelle élémentaire mesurait $20 \mathrm{~m} \times 6,4 \mathrm{~m}$ pour une surface utile de $20 \mathrm{~m} \times 3,2 \mathrm{~m}$ (4 lignes de 20 mètres). Huit champs fertiles et huit champs dégradés ont été mis en place à Gashiga comme à Ngong.

L'objectif était de comparer l'impact sur la production agricole de niveaux d'intensification croissants de la culture du maïs par rapport à l'itinéraire technique généralement pratiqué par le producteur. Le témoin $(\mathrm{CP})$ était donc conduit par le producteur lui-même, sans intervention de notre part. À contrario, les niveaux d'intensification NI1 et NI2 étaient mis en place par la recherche selon des itinéraires préétablis décrits dans le Tableau 1. Le premier niveau d'intensification (NI1) correspondait aux préconisations de la fiche technique actuellement diffusée par l'encadrement d'après les résultats de la recherche agronomique au Nord Cameroun, sauf en ce qui concerne la densité visée qui était une densité intermédiaire entre la densité généralement observée chez les agriculteurs et la recommandation de la fiche technique. Le second niveau d'intensification (NI2) était une intensification par rapport au niveau NI1 concernant le traitement des semences, la densité de culture visée, la maîtrise de l'enherbement et la fumure organo-minérale.

Les itinéraires intensifs correspondant à NI1 et NI2 sont faisables au Nord Cameroun, les intrants et appareils nécessaires à leur réalisation étant proposés aux producteurs par l'encadrement. Par contre, il n'a pas été tenu compte dans l'élaboration du protocole d'expérimentation de la contrainte des ressources financières et du supplément de main-d'œuvre requis pour leur mise en place ; ces aspects feront l'objet de la discussion. 
Tableau 1. Description des deux niveaux d'intensification testés au Nord Cameroun, campagne 2013. Table 1. Description of the two levels of intensification tested in northern Cameroon, crop year 2013.

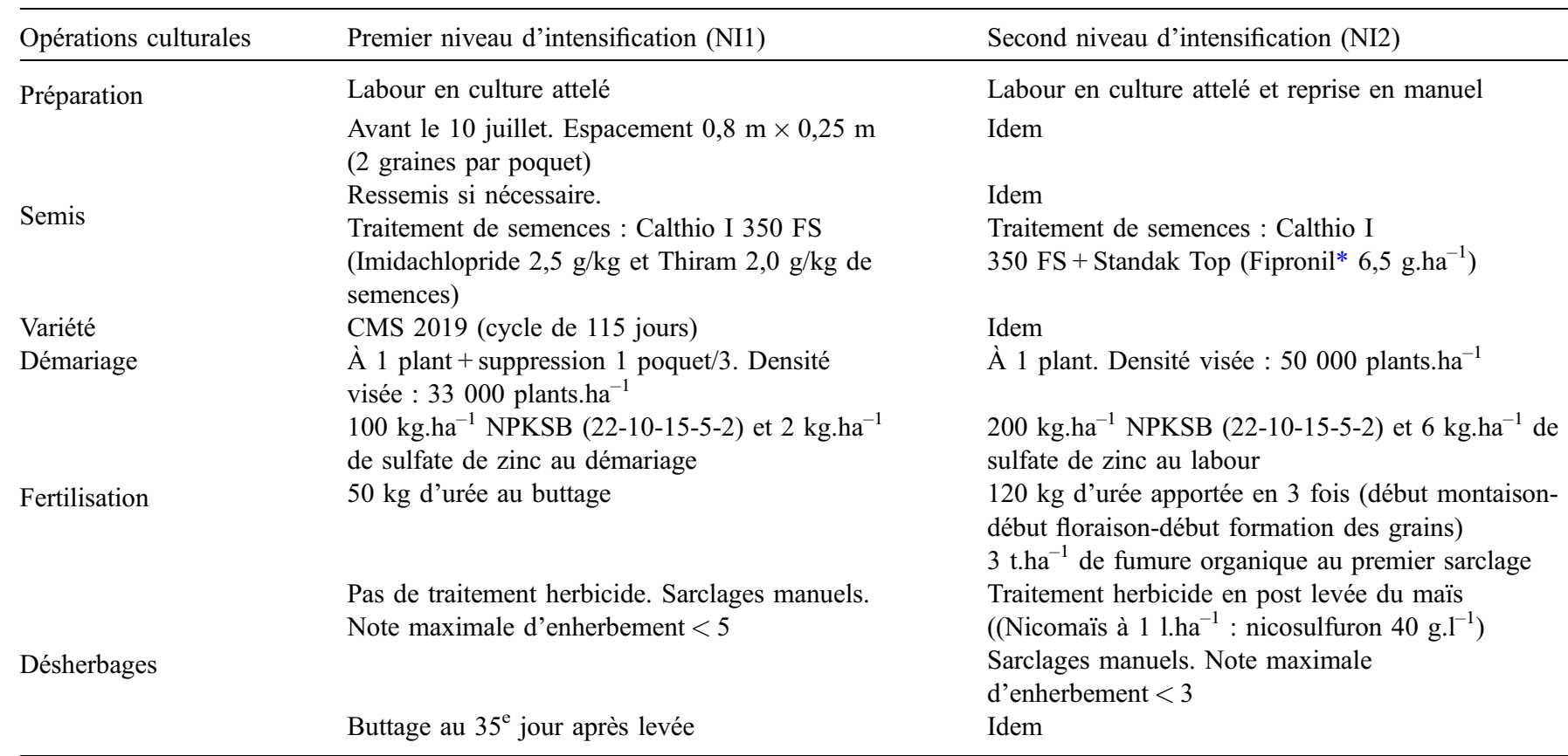

\footnotetext{
* Il est à noter que le Fipronil fait l'objet de restrictions d'usage par l'Union européenne depuis 2013 en raison de sa forte toxicité suspectée envers les abeilles et les insectes pollinisateurs. Il est toujours autorisé au Cameroun en 2016. Il conviendra néanmoins de proposer à l'avenir des traitements de semences moins sujets à controverse quant à leur impact sur l'environnement.
}

\subsection{Les observations et les mesures réalisées}

L'itinéraire technique réalisé sur chaque parcelle élémentaire a été relevé. Des comptages et observations ont été faits sur les états culturaux : densité, cotations d'enherbement de 1 (enherbement nul) à 9 (enherbement total) selon la méthode décrite par Mathieu et Marnotte (2000), nombre de plants attaqués par Striga hermonthica, nombre de plants versés à la récolte. Les rendements parcellaires ont été obtenus par la récolte en épis des parcelles utiles, leur séchage, leur égrenage et la pesée du maïs-grain.

\subsection{Les analyses statistiques}

L'analyse des résultats a suivi la méthode décrite par Cochran et Cox (1957: p. 545-550) pour l'analyse d'une série d'essais à dispositif identique. Chaque champ était considéré comme un lieu. Une analyse de variance sur les résultats de chaque lieu, avec deux répétitions, a permis d'extraire les moyennes par modalité de niveaux d'intensification et la variance résiduelle. Puis ces moyennes ont fait l'objet d'une nouvelle analyse de variance du regroupement des lieux permettant de tester l'existence d'un effet du niveau d'intensification et d'une interaction entre niveau d'intensification et lieu.

\subsection{Les itinéraires techniques réels observés}

Le suivi des parcelles montre un premier écart au protocole avec des dates de semis moyennes tardives pour les modalités NI1 et NI2. Quatre champs à Ngong et six à Gashiga ont été semés après le 10 juillet qui est une date à ne pas dépasser pour réaliser le semis du maïs en référence aux recommandations. Par chance, la pluviosité de fin de saison a été bonne sur les deux sites, se prolongeant jusqu'à la seconde décade d'octobre, si bien que les cycles des cultures se sont déroulés normalement même en conditions de semis tardifs. Un second écart à noter est la densité moyenne à la récolte de la modalité NI1 qui s'avère inférieure à celle prévue dans le protocole et qui au final est similaire en moyenne à celle de la modalité CP (Tab. 2). On peut noter aussi le choix presque généralisé par les producteurs du recours aux herbicides ainsi que la faible fréquence d'utilisation de la fumure organique sur le traitement $\mathrm{CP}$. De façon générale, les itinéraires techniques des producteurs (CP) et du premier niveau d'intensification (NI1) sont assez proches en termes d'utilisation des engrais minéraux.

\subsection{Les analyses économiques}

Pour comparer la rentabilité économique des niveaux d'intensification étudiés, nous avons utilisé comme indicateur la valorisation de la journée de travail. Pour ce faire, il fallait estimer les coûts de production à partir des coûts des intrants, estimer les temps de travaux et une valeur du prix d'achat aux producteurs du maïs-grain.

Les prix de cession des intrants figurent au Tableau 3. À partir de ces données, le coût moyen total des intrants de chaque itinéraire a été calculé, par type de parcelle et par site en référence aux relevés des pratiques culturales (Tab. 4). 
Tableau 2. Opérations culturales réalisées et états culturaux observés sur les champs d'essais intensification du maïs, Nord Cameroun, campagne 2013.

Table 2. Cultivation practices and crop conditions observed on tests fields during Maize Culture Intensification, North Cameroon, crop year 2013.

\begin{tabular}{|c|c|c|c|}
\hline Opération ou état cultural & \multicolumn{3}{|c|}{ Modalités comparées } \\
\hline$\%$ de parcelles labourées & 94 & 100 & 100 \\
\hline Date de semis moyenne & 07/07/2013 & $08 / 07 / 2013$ & 08/07/2013 \\
\hline$\%$ de parcelles avec traitement de semences & 25 & 0 & 100 \\
\hline$\%$ de parcelles avec ressemis & 9 & 59 & 59 \\
\hline Nombre moyen de désherbages*** & 1,9 & 1,9 & 2,8 \\
\hline$\%$ de parcelles avec amendement organique & 6 & 0 & 100 \\
\hline Délais moyen d'épandage de l'urée**** (JAS) & 35 & 44 & 25 \\
\hline$\%$ de parcelles buttées & 66 & 94 & 94 \\
\hline Dose moyenne d'engrais complet NPKSB $\left(\mathrm{kg} \cdot \mathrm{ha}^{-1}\right)$ & 71 & 100 & 200 \\
\hline Dose moyenne d'urée $\left(\mathrm{kg} \cdot \mathrm{ha}^{-1}\right)$ & 50 & 50 & 120 \\
\hline Densité à la levée (nombre de plants.ha ${ }^{-1}$ ) & 30600 & 29100 & 41800 \\
\hline Densité à la récolte (nombre de plants.ha ${ }^{-1}$ ) & 27400 & 28000 & 40000 \\
\hline Verse à la récolte (nombre de plants.ha ${ }^{-1}$ versés) & 2400 & 1200 & 1900 \\
\hline
\end{tabular}

${ }^{*} \mathrm{CP}$ : conduite par producteur ; NI1 : premier niveau d'intensification ; NI2 : second niveau d'intensification.

${ }^{* *}$ JAS : nombre de jours après semis (avant semis si négatif).

*** Mécaniques et chimiques.

***** Pour NI2 : délais moyen du premier épandage d'urée.

Tableau 3. Coût des intrants utilisés sur les différents itinéraires techniques testés, essai intensification du maïs, Nord Cameroun, campagne 2013 (1 $000 \mathrm{FCFA}=1,524 €)$.

Table 3. Cost of inputs used in different cultivation systems tested, Maize Intensification test, North Cameroon, 2013 crop year. (1 000 FCFA $=1.524 €)$.

\begin{tabular}{llcl}
\hline Intrant & Unité & Coût par unité (FCFA) & Fournisseur \\
\hline Engrais complet & & 392 & SODECOTON \\
Urée & $\mathrm{kg}$ & 392 & SODECOTON \\
Sulfate de zinc & & 1100 & SODECOTON \\
Semences certifiées & & 800 & IRAD \\
Semences ordinaires & Litre & 140 & Producteurs \\
Herbicide maïs & Tonne & 6000 & SODECOTON \\
Amendement organique & Pour $15 \mathrm{~kg}$ de semences & 3000 & Producteurs \\
Traitement semences NI1* & Pour $30 \mathrm{~kg}$ de semences & 82000 & Produit non vulgarisé. Prix calculé \\
Traitement semences NI2* & & & avec coût des constituants \\
& &
\end{tabular}

\footnotetext{
* Voir légende Tableau 2.
}

Pour déterminer les besoins en temps de travail selon les niveaux d'intensification, nous avons retenu les références disponibles dans la littérature car l'évaluation de ces temps ramenés à l'hectare n'était pas réalisable sur les parcelles d'essai vu leur petite taille : Djamen et al. (2015), Coulibaly et al. (2012), Guibert et al. (2003), Adegbidi (1995), Faure et al. (1995). Pour le niveau CP, un investissement moyen en travail de 85 homme-jour.ha $^{-1}$ est nécessaire, du labour à la récolte (Tab. 5).
Le prix d'achat du maïs aux producteurs n'est pas aisé à déterminer, car il n'est pas fixé pour toute la période de commercialisation et fluctue dans l'année, d'une année sur l'autre et en fonction de la proximité du lieu de production par rapport aux centres de consommation (Fig. 3). Le prix idoine à prendre en considération ne peut être la moyenne annuelle des prix relevés, d'une part parce qu'il faudrait pouvoir pondérer ceux-ci par les quantités effectivement échangées et d'autre part parce qu'il faut considérer un prix auquel le 
Tableau 4. Calcul du coût moyen en intrants des différents itinéraires techniques testés, essai intensification du maïs, Nord Cameroun, campagne $2013(1000$ FCFA $=1,524 €)$.

Table 4. Calculating the cost of inputs of cultivation systems tested, Maize Intensification test, North Cameroon, $2013 \mathrm{crop}$ year. $(1000$ FCFA = $1.524 €)$.

\begin{tabular}{|c|c|c|c|c|c|c|c|}
\hline \multirow{2}{*}{ Intrants } & \multirow{2}{*}{$\begin{array}{l}\text { Doses ou fréquences de } \\
\text { réalisation et coûts moyens }\end{array}$} & \multicolumn{4}{|c|}{ Conduite producteur } & \multirow{2}{*}{ NI1* } & \multirow{2}{*}{ NI2* } \\
\hline & & Fertiles & Dégradées & Fertiles & Dégradées & & \\
\hline \multirow[b]{2}{*}{ Engrais complet } & Dose $\left(\mathrm{kg} \cdot \mathrm{ha}^{-1}\right)$ & 72 & 63 & 63 & 84 & 100 & 200 \\
\hline & Coût (FCFA) & 28300 & 24800 & 24900 & 33190 & 39300 & 78600 \\
\hline Urée & Dose $\left(\mathrm{kg} \cdot \mathrm{ha}^{-1}\right)$ & 55 & 40 & 55 & & 50 & 120 \\
\hline $\mathrm{ZnSO}_{4}$ & Coût (FCFA) & 0 & 0 & 2750 & 490 & 2200 & 6600 \\
\hline \multirow{2}{*}{ Matière organique } & Fréquence & 0,25 & 0 & 0 & 0 & 0 & 1 \\
\hline & Coût (FCFA) & 2230 & 0 & 0 & 0 & 0 & 9000 \\
\hline Semences & Coût (FCFA) & 2100 & 2100 & 2100 & 2100 & 12000 & 24000 \\
\hline Traitement semences & Fréquence & 0,54 & 0 & 0 & 0,44 & 1 & 1 \\
\hline
\end{tabular}

* Voir légende Tableau 2.

Tableau 5. Estimations des temps de travaux sur les champs d'essais intensification du maïs, Nord Cameroun, campagne 2013.

Table 5. Estimates of work time on test fields of Maize Intensification, North Cameroon, 2013 crop year.

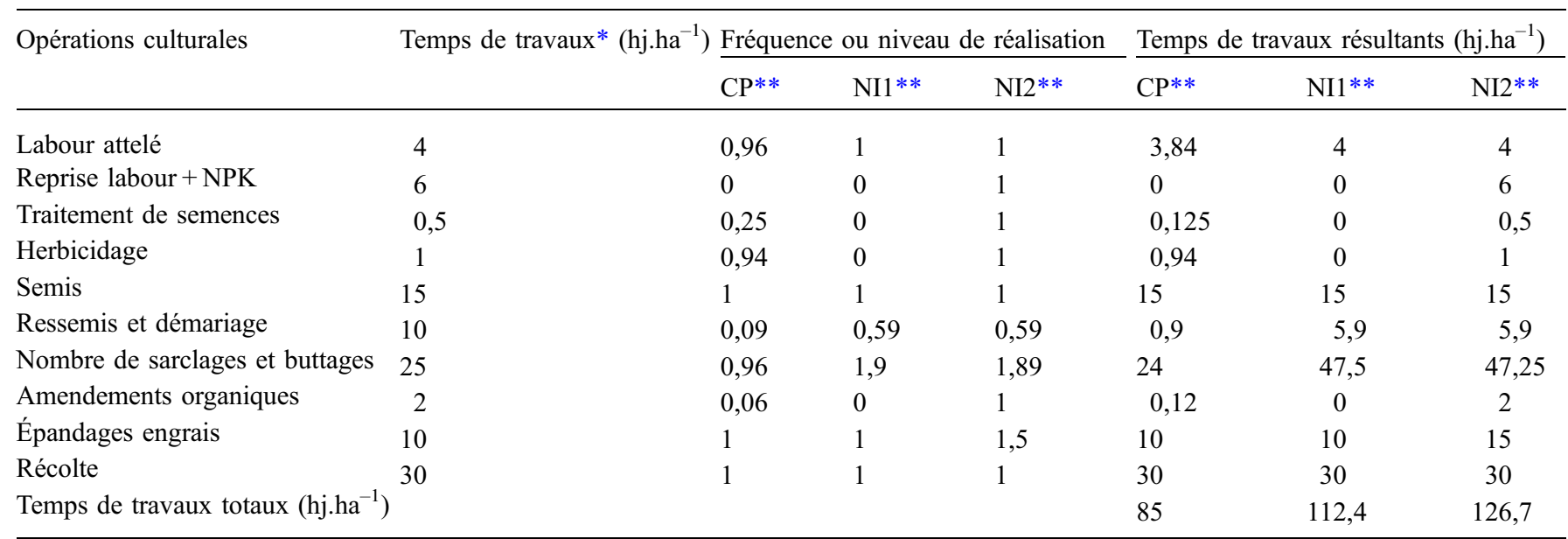

${ }_{*}^{*}$ D'après la littérature hj $=$ homme-jour. hj $=$ homme-jour.
${ }^{* *}$ Voir légende Tableau 2 .

producteur peut réellement compter vendre sa production avant la mise en place de la campagne. Au vu de ces différents éléments, le prix d'achat de 120 FCFA. $\mathrm{kg}^{-1}$ rendu Garoua est un prix auquel le producteur peut espérer vendre sa production dans $80 \%$ des cas. En prenant en compte le coût du transport, un prix de $100 \mathrm{FCFA} . \mathrm{kg}^{-1}$ bord champ a été retenu. Coulibaly et al. (2012) et Djamen et al. (2015) ont utilisé un prix de respectivement 125 et 110 FCFA. $\mathrm{kg}^{-1}$ sans cependant en préciser les contours. À partir de ces données, il est possible de faire des projections en simulant différents scénarios de prix.

\section{Résultats}

Des accroissements significatifs de rendement sont obtenus par le niveau de fertilité des sols et l'intensification des cultures, sans rendre cette intensification profitable.

\subsection{Analyse des rendements}

L'analyse de la variance du regroupement sur l'ensemble des lieux de chacun des deux sites donne les résultats figurant au Tableau 6. 


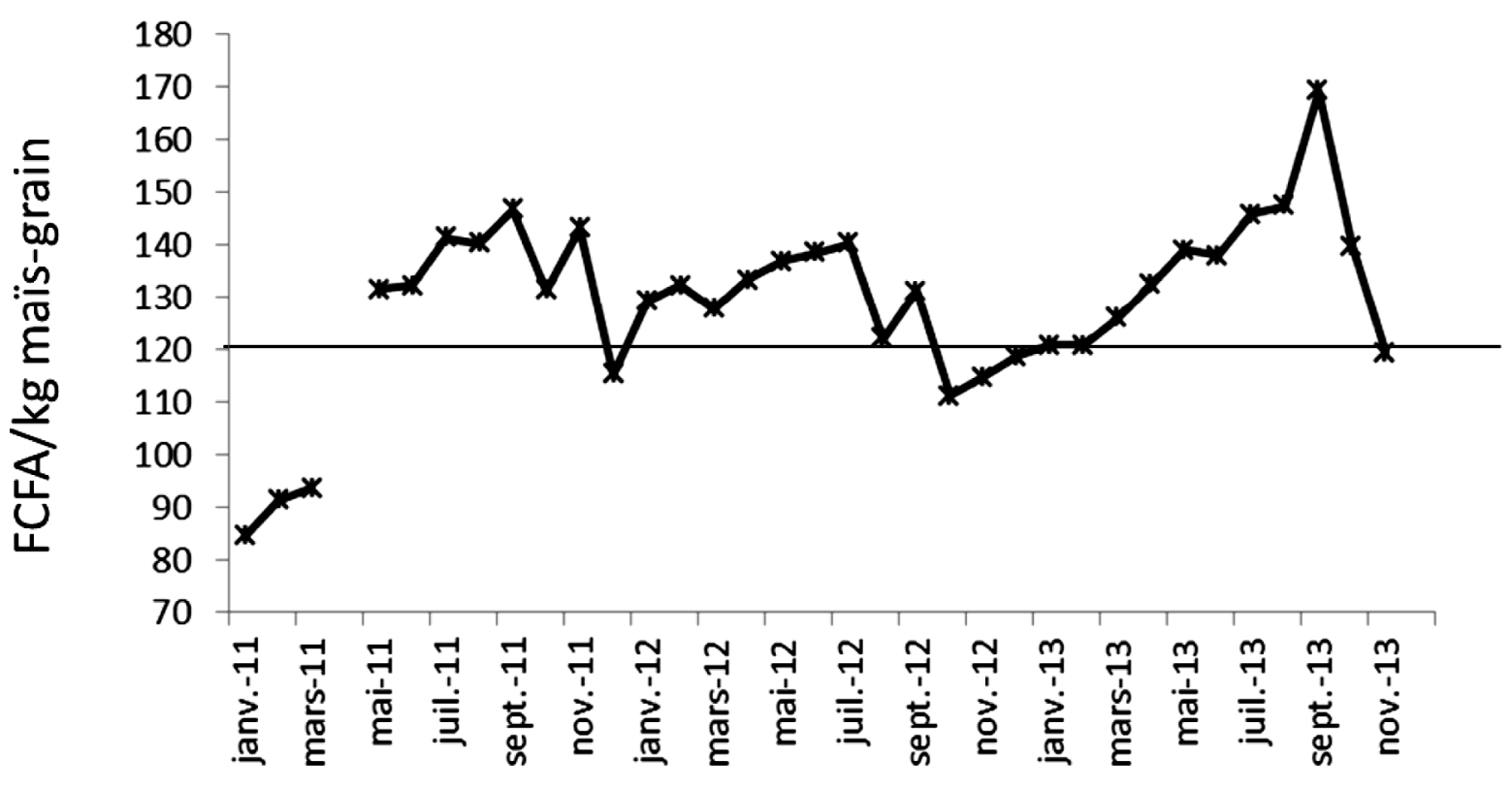

\section{Mois}

Fig. 3. Mercuriales du prix d'achat du maïs au producteur à Garoua, nord Cameroun, entre 2011 et $2013(1000 \mathrm{FCFA}=1,524 €)$. Fig. 3. Mercurial of corn purchase price from the producer at Garoua, Northern Cameroon between 2011 and $2013(1000$ FCFA $=\epsilon 1.524)$. Source : mercuriales de l'office céréalier de Garoua.

Tableau 6. Analyse de la variance du regroupement par groupes de champs homogènes, essai intensification du maïs, Nord Cameroun, campagne 2013.

Table 6. Analysis of the variance of the combination of fields by homogeneous groups of fields for maize intensification test, Northern Cameroon, crop year 2013.

\begin{tabular}{lllll}
\hline Sites & Modalités & $\begin{array}{l}\text { Moyennes rendements } \\
\text { maïs-grain }\left(\mathrm{kg}^{-1} \mathrm{ha}^{-1}\right)\end{array}$ & $\begin{array}{l}\text { Signification Niveaux } \\
\text { intensification }\end{array}$ & $\begin{array}{l}\text { Signification Interaction Niveaux } \\
\text { intensification X Champs }\end{array}$ \\
\hline \multirow{3}{*}{ Ngong } & Conduite producteur & $1550 \mathrm{c}$ & & $\mathrm{S}(\mathrm{F}=2,0)$ \\
& Premier niveau intensification & $2123 \mathrm{~b}$ & $\mathrm{HS}(\mathrm{F}=45,1)$ & \\
& Second niveau intensification & $3059 \mathrm{a}$ & & $\mathrm{S} * *(\mathrm{~F}=1,9)$ \\
Gashiga & Conduite producteur & $1646 \mathrm{~b}$ & $\mathrm{HS} *(\mathrm{~F}=73,2)$ & \\
& Premier niveau intensification & $1937 \mathrm{~b}$ & & \\
\hline
\end{tabular}

\footnotetext{
${ }^{*} \mathrm{HS}=$ différences entre les moyennes significatives par le test de Newman-Keuls au seuil de probabilité de 0,01.

${ }^{* *} \mathrm{~S}=$ différences entre les moyennes significatives par le test de Newman-Keuls au seuil de probabilité de 0,05 ; les moyennes suivies d'une même lettre ne sont pas significativement différentes par le même test au seuil de probabilité de 0,05 .
}

L'interaction facteur étudié (niveau d'intensification) X lieu étant significative pour chacun des deux sites, les résultats ne peuvent pas être interprétés globalement par site.

Une partition par groupes homogènes qui parait plausible est de prendre en compte le niveau de fertilité des champs (Tab. 7). Quatre groupes homogènes sont obtenus. Les résultats des analyses de la variance du regroupement sur ces quatre groupes indiquent que les interactions facteur étudié X lieux ne sont plus significatives.

Les rendements obtenus avec les niveaux CP et NI1 ne sont pas différents, sauf pour les parcelles dégradées du site de Ngong. Le rendement obtenu avec le niveau d'intensification NI2 est toujours significativement supérieur à celui des deux autres modalités pour les quatre groupes, avec une augmentation en moyenne d'environ $1,0 \mathrm{t}^{\mathrm{ha}^{-1}}$ à Ngong (pluviosité médiocre) et 1,5 t.ha $^{-1}$ à Gashiga (meilleure pluviosité), et ce de façon uniforme pour les champs dégradés comme fertiles.

\subsection{Analyses économiques}

Les résultats de l'analyse économique avec un prix d'achat au producteur du maïs-grain de 100 FCFA.kg ${ }^{-1}$ figurent au Tableau 8. Le coût total des intrants des parcelles CP et NI1 est proche. Il est multiplié en moyenne par un facteur de 2,4 en passant de la modalité NI1 à NI2 du fait principalement d'un apport 
Tableau 7. Analyse de la variance du regroupement des champs par sites, essai intensification du maïs, Nord Cameroun, campagne 2013. Table 7. Analysis of the variance of the combination of fields by testing sites for maize intensification, Northern Cameroon, crop year 2013.

\begin{tabular}{|c|c|c|c|c|}
\hline Groupes & Modalités & 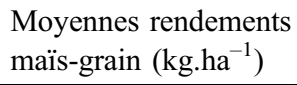 & $\begin{array}{l}\text { Signification Niveaux } \\
\text { intensification }\end{array}$ & $\begin{array}{l}\text { Signification Interaction Niveaux } \\
\text { intensification X Champs }\end{array}$ \\
\hline & Conduite producteur & $2430 \mathrm{~b}$ & & \\
\hline \multirow{2}{*}{ Ngong champs fertiles } & Second niveau intensification & 3426 a & \multirow{2}{*}{$\mathrm{HS}^{*}(\mathrm{~F}=12,2)$} & \multirow{2}{*}{$\mathrm{NS}^{*}(\mathrm{~F}=1,5)$} \\
\hline & Conduite producteur & $843 \mathrm{c}$ & & \\
\hline Ngong champs dégradés & Premier niveau intensification & $1563 \mathrm{~b}$ & HS $(F=58,2)$ & NS $(F=0,8)$ \\
\hline \multirow[t]{3}{*}{ Gashiga champs fertiles } & Premier niveau intensification & $2247 \mathrm{~b}$ & \multirow[t]{3}{*}{$\mathrm{HS}(\mathrm{F}=56,2)$} & \multirow[t]{3}{*}{$\mathrm{NS}(\mathrm{F}=1,1)$} \\
\hline & Second niveau intensification & 3715 a & & \\
\hline & Conduite producteur & $1217 \mathrm{~b}$ & & \\
\hline \multirow[t]{2}{*}{ Gashiga champs dégradés } & Premier niveau intensification & $1474 \mathrm{~b}$ & \multirow[t]{2}{*}{$\mathrm{HS}(\mathrm{F}=36,4)$} & \multirow[t]{2}{*}{$\mathrm{NS}(\mathrm{F}=1,7)$} \\
\hline & Second niveau intensification & $3021 \mathrm{a}$ & & \\
\hline
\end{tabular}

${ }^{*}$ HS : voir légende Tableau 6 ; NS = différences entre les moyennes non significatives par le test de Newman-Keuls au seuil de probabilité de $0,05$.

Tableau 8. Résultats économiques des différents itinéraires techniques, essai intensification du maïs, Nord Cameroun, campagne 2013 $(1000$ FCFA = 1,524 €).

Table 8. Economic results for various cultural practices, maize intensification tests, Northern Cameroon, crop year 2013.

\begin{tabular}{|c|c|c|c|c|c|c|}
\hline Sites & Types de champs & Modalités* & $\begin{array}{l}\text { Coûts des intrants } \\
\left(\text { FCFA.ha }{ }^{-1}\right)\end{array}$ & $\begin{array}{l}\text { Produits } \\
\left(\text { FCFA.ha }^{-1}\right)\end{array}$ & $\begin{array}{l}\text { Temps de } \\
\text { travaux }\left(\mathrm{hj} \cdot \mathrm{ha}^{-1}\right)\end{array}$ & $\begin{array}{l}\text { Valorisation de la journée } \\
\text { de travail (FCFA.j }{ }^{-1} \text { ) }\end{array}$ \\
\hline \multirow[b]{3}{*}{ Ngong } & \multirow{2}{*}{ Fertiles } & $\mathrm{CP}$ & 62000 & 243000 & 85 & 2130 \\
\hline & & NI2 & 181140 & 342600 & 127 & 1280 \\
\hline & Dégradés & $\mathrm{CP}$ & 62580 & 84300 & 85 & 720 \\
\hline \multirow{5}{*}{ Gashiga } & \multirow{3}{*}{ Fertiles } & $\mathrm{CP}$ & 57960 & 215900 & 85 & 1860 \\
\hline & & NI1 & 75900 & 224700 & 112 & 1320 \\
\hline & & NI2 & 181140 & 371500 & 127 & 1500 \\
\hline & \multirow[b]{2}{*}{ Dégradés } & $\mathrm{CP}$ & 47600 & 121700 & 85 & 700 \\
\hline & & NI1 & 75900 & 147400 & 112 & 640 \\
\hline
\end{tabular}

\footnotetext{
* Voir légende Tableau 2.
}

bien supérieur d'engrais minéral pour le niveau d'intensification le plus fort. Les valorisations des journées de travail sont faibles sur champs dégradés sur les deux sites, en moyenne de 560 FCFA.j $^{-1}$ et trois fois plus importantes sur sols fertiles. Les meilleures valorisations des journées de travail sont obtenues avec l'itinéraire $\mathrm{CP}$, en moyenne de 1385 FCFA.j ${ }^{-1}$. Les valorisations moyennes sur parcelles NI2 sont inférieures à celles des parcelles $\mathrm{CP}$, sauf sur le site de Ngong pour les parcelles dégradées où elles sont équivalentes, et sur parcelles fertiles à Gashiga.

Les Figures 4 et 5 présentent les simulations d'augmentation du prix d'achat du maïs au producteur, les prix des intrants restants constants. Au prix d'achat au producteur de $60 \mathrm{FCFA} . \mathrm{kg}^{-1}$, prix possible au moment de la récolte et en cas de forte production régionale, la valorisation de la journée de travail est négative ou quasi-nulle partout, sauf pour les parcelles fertiles sous NI1 sur les deux sites, où elle atteint environ $500 \mathrm{FCFA}^{-1}$. Le niveau d'intensification NI2 est plus rentable que le NI1 sur parcelles dégradées à partir d'un prix d'achat de 70 et 115 FCFA. $\mathrm{kg}^{-1}$, respectivement sur les sites de Gashiga et Ngong. Sur parcelles fertiles, NI2 ne dépasse pas en rentabilité NI1 à Ngong pour un prix inférieur à 130 FCFA. $\mathrm{kg}^{-1}$. Sur le site de Gashiga et sur parcelles fertiles, NI2 devient plus rentable que NI1 à partir d'un prix de 80 FCFA. $\mathrm{kg}^{-1}$ et procure un surplus de 600 FCFA.j $^{-1}$ avec un prix de 120 FCFA. $\mathrm{kg}^{-1}$.

\section{Discussion}

Les rendements du maïs sont impactés négativement par le niveau de dégradation des sols, quel que soit l'itinéraire pratiqué, et positivement par le niveau d'intensification, quel que soit le niveau de fertilité. Malgré ces résultats, l'intensification renforcée de la culture du maïs n'est pas profitable pour les producteurs aux prix actuels. Elle le devient à partir d'un prix d'achat du maïs-grain au producteur de 100 FCFA. $\mathrm{kg}^{-1}$ bord champ sur le site de Gashiga. 


\section{Valorisation de la journée de travail (FCFA)}

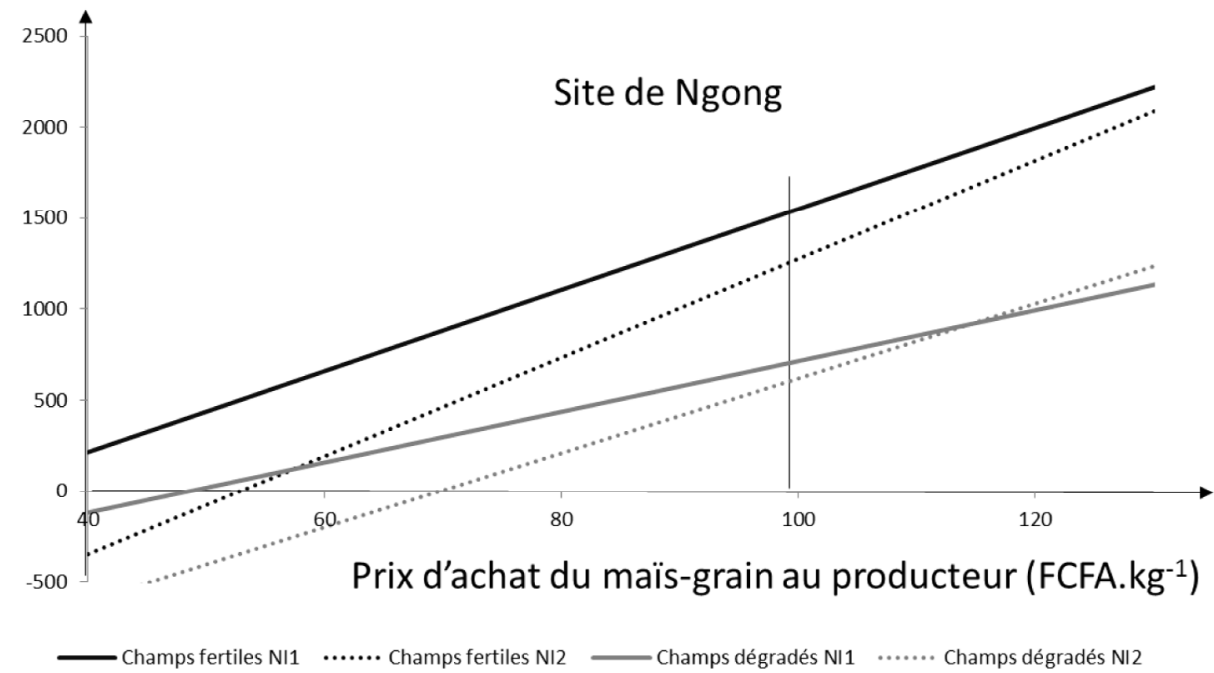

\section{NI1 : premier niveau d'intensification NI2 : second niveau d'intensification}

Fig. 4. Simulation de scénarios d'augmentation du prix d'achat du kilo de maïs sur le site de Ngong sous différents itinéraires techniques (1000 FCFA = 1,524 €).

Fig. 4. Simulation scenarios to increase the purchase price of a kilogram of maize on the Ngong site under different agricultural practices (1 000 FCFA $=€ 1.524)$.

\section{Valorisation de la journée de travail (FCFA)}

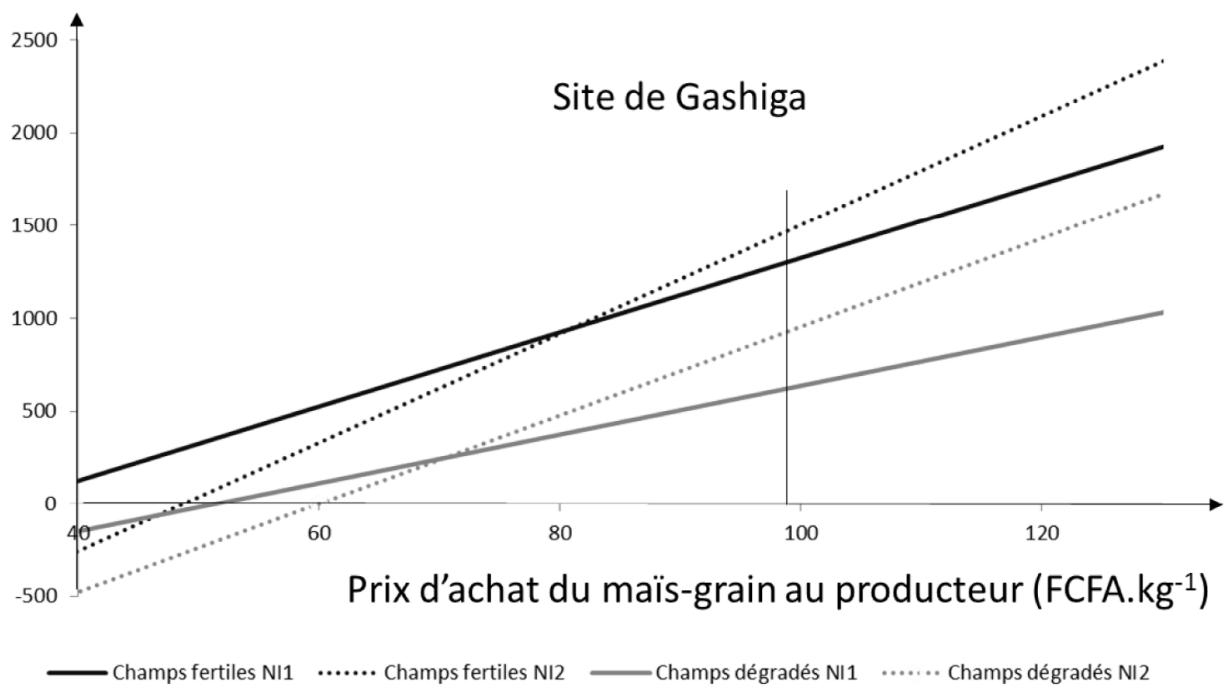

\section{NI1 : premier niveau d'intensification NI2 : second niveau d'intensification}

Fig. 5. Simulation de scénarios d'augmentation du prix d'achat du kilo de maïs sur le site de Gashiga sous différents itinéraires techniques $(1000 \mathrm{FCFA}=1,524 €)$.

Fig. 5. Simulation scenarios to increase the purchase price of a kilogram of maize on the Gashiga site under different agricultural practices (1 000 FCFA $=€ 1.524)$.

\subsection{Effets de la dégradation des sols et de l'intensification sur les rendements du maïs}

Le niveau de rendement moyen des parcelles $\mathrm{CP}$ est de $1662 \mathrm{~kg} \cdot \mathrm{ha}^{-1}$, proche des rendements moyens observés et rapportés dans la littérature (entre 1550 et $1950 \mathrm{~kg} \cdot \mathrm{ha}^{-1}$, voir supra). Les rendements obtenus sur les parcelles CP et NI1 sont assez proches, sauf sur champs dégradés à Ngong. Les producteurs conduisent leurs parcelles de maïs selon un itinéraire technique proche de la fiche technique vulgarisée, ce 
qui explique la proximité entre les niveaux de rendement obtenus pour ces deux types de parcelles. Les rendements des parcelles NI2 sont toujours significativement supérieurs à ceux des parcelles NI1. Cette différence est plus importante sur le site de Gashiga que sur celui de Ngong. L'hypothèse qui peut être faite est que les résultats techniques de l'intensification sont meilleurs avec une pluviosité plus importante. Smith (1995) indique combien la culture du maïs est sensible aux stress hydriques, notamment pendant la phase de maturité. Drury et al. (1994) montrent l'importance de la maitrise de l'alimentation hydrique de la culture de maïs sur l'efficience de l'azote.

Par contre, le niveau de fertilité des champs n'impacte pas les gains de rendement obtenus par l'intensification du niveau NI2 par rapport au niveau NI1. Les champs dégradés de l'étude sont néanmoins cultivés par les producteurs pour une culture qu'ils savent exigeante. L'état de dégradation de ces champs est encore relatif et un niveau élevé de fertilisants ainsi qu'une bonne maîtrise des mauvaises herbes, apportés par l'intensification, peuvent encore compenser un état de fertilité plus faible. Les résultats ne peuvent pas être étendus à des états de dégradation plus forts qui n'ont pas été étudiés ici.

L'établissement d'une carte de la région délimitant les zones favorables à une intensification plus poussée de la culture du maïs serait un outil profitable pour un projet d'amélioration de la sécurité alimentaire au Nord Cameroun. Il pourrait se réaliser par l'extension de cette expérimentation sur un plus grand nombre de sites et d'années, englobant ainsi une gamme plus importante de niveaux de fertilité et de conditions hydriques. Le recours à des indicateurs de fertilité des sols et à des simulations de bilan hydrique pourraient accompagner cette étude.

\subsection{Rentabilité de l'intensification de la culture du maïs}

L'impact du niveau de fertilité des champs sur la valorisation du travail est constant et important, la différence de la valorisation de la journée de travail étant en moyenne de plus de 1000 FCFA en faveur des champs fertiles pour un prix d'achat au producteur de 100 FCFA. $\mathrm{kg}^{-1}$. La dégradation des champs induit un manque à gagner moyen de près de 80000 FCFA.ha ${ }^{-1}$. Au niveau des prix actuels d'achat du maïs (fourchette de prix 60-120 FCFA. kg ${ }^{-1}$ ), l'intensification accrue de la culture du maïs ne s'avère pas rentable pour le producteur de Ngong, site et année caractérisés par une pluviométrie déficitaire. Par contre, elle est rentable à Gashiga à partir d'un prix d'achat supérieur à $100 \mathrm{FCFA} . \mathrm{kg}^{-1}$, à la fois en termes de rémunération du travail et de retour sur investissement pour les intrants. De fait, seules les exploitations ayant bénéficié de conditions pluviométriques satisfaisantes et surtout pouvant valoriser correctement leur maïs, notamment en évitant de le vendre pendant la période suivant immédiatement les récoltes où les prix sont généralement bas, peuvent tirer profit d'une intensification accrue de la culture du maïs. C'est d'ailleurs ce qui est constaté dans certaines exploitations de type entrepreneurial, qualifiées d' "émergentes » par la société cotonnière, qui à la fois intensifient leur culture de maïs et maîtrisent mieux la valorisation de leur production en organisant leur commercialisation, parfois directement jusque vers la capitale (Guibert et al., 2015).

\subsection{Intensifier sans augmenter le temps de travail pendant la mise en place des cultures ?}

L'itinéraire des producteurs (CP) se caractérise par une mise en place rapide de la culture : peu ou pas de traitements de semences, de ressemis ou de démariage et une utilisation généralisée de l'herbicide pour gagner du temps. La logique des producteurs est de mettre en place le plus rapidement leurs parcelles pour s'affranchir d'aléas climatiques. A contrario, l'état cultural résultant de cette pratique, en particulier la densité de culture, n'est pas propice à une intensification poussée, notamment par les intrants. Or, le niveau d'intensification proposé (NI2) augmente le temps de travail et notamment celui lié à la mise en place de la culture. Pour résoudre cette opposition, il faudrait concevoir des outils et pratiques capables à la fois de ne pas augmenter le temps de mise en place des cultures et d'assurer un état cultural propice à l'intensification, comme l'utilisation de semoir en semis direct par exemple.

Ces résultats économiques reposent sur la détermination de temps de travaux qui sont peu documentés, du fait des difficultés à les mesurer, et qui sont par nature assez peu robustes, étant donné qu'ils sont susceptibles de variations d'une exploitation à l'autre. Cependant, le calcul de la valorisation de la journée de travail est le meilleur indicateur de la rentabilité d'un itinéraire technique dans un milieu où c'est la force de travail qui conditionne le niveau de production.

\section{Conclusion}

Malgré les résultats techniques significatifs obtenus par un niveau d'intensification élevé de la culture du maïs, avec des gains de 1 à 1,5 t.ha ${ }^{-1}$ par rapport aux pratiques actuelles des producteurs, les résultats économiques sont peu favorables pour cette pratique du fait des prix actuels des intrants et des prix d'achat du maïs au producteur. L'étude montre que la dégradation des sols a un impact économique considérable sur le revenu des producteurs. Toutefois, l'hypothèse peut être faite que les conditions climatiques impactent également les résultats de l'intensification. L'intensification du maïs par augmentation des doses d'intrants pourra avoir lieu si les prix agricoles augmentent de façon durable, ce qui entre en contradiction avec une sécurité alimentaire accrue pour les populations vulnérables. La solution de lutter contre l'appauvrissement des terres reste celle qui peut à la fois procurer une augmentation de revenus pour les producteurs et répondre au problème de la sécurité alimentaire de l'ensemble des régions et pays concernés.

\section{Références}

Adegbidi AA. 1995. Les performances du maïs amélioré TZB en milieu paysan dans le département de l'Atacora au Bénin. In : Production et valorisation du maïs à l'échelon villageois en Afrique de l'Ouest: actes du séminaire "Maïs prospère», 25-28 janvier 1994. Montpellier (France): CIRAD-SAR, UNBFSA.

Brabant P, Gavaud M. 1985. Les sols et les ressources en terre du Nord-Cameroun (Provinces du Nord et de l'Extrême-Nord). Bondy (France): ORSTOM. 
Cochran WR, Cox GM. 1957. Experimental Designs, 2nd ed. NewYork: J. Wiley.

Coulibaly K, Vall E, Autfray P, Sedogo MP. 2012. Performance technico-économique des associations maïs/niébé et maïs/mucuna en situation réelle de culture au Burkina Faso : potentiels et contraintes. Tropicultura 30(3): 147-154

Djamen Nana P, Andrieu N, Zerbo I, Ouédraogo Y, Le Gal PY. 2015. Agriculture de conservation et performances des exploitations agricoles en Afrique de l'Ouest. Cahiers Agricultures 24: 113-122 doi: http://dx.doi.org/10.1684/agr.2015.0743

Drury CF, Tan CS, Gaynor JD, Oloya TO, Welacky TW. 1994. Influence of controlled drainage/sub irrigation on nitrate loss from Brookston clay loam soil. St Joseph (USA): American Society of Agricultural Engineers, p. 1-13.

Faure G, Fusillier J-L, Marchand J-L. 1995. Les effets de la mécanisation sur la productivité en maïsiculture. Cas de la zone cotonnière. In : Production et valorisation du maïs à l'échelon villageois en Afrique de l'Ouest: actes du séminaire "Mä̈s prospère », 25-28 janvier 1994. Montpellier (France): CIRADSAR, UNB-FSA.

Fusillier J-L. 1995. Bilan et perspectives de la diffusion de la maïsiculture en zone de savane d'Afrique de l'Ouest. In : Production et valorisation du maïs à l'échelon villageois en Afrique de l'Ouest : actes du séminaire "Maïs prospère », 25-28 janvier 1994. Montpellier (France): CIRAD-SAR, UNB-FSA.

Guibert H, Njiti CF, Labonne M, Njoya A, Gautier D, Mbouyo E, et al. 2003. Comparaison de la productivité des principales activités rurales au Nord-Cameroun. In : Jamin JY, Seiny Boukar L, Floret C (ed), Savanes africaines : des espaces en mutation, des acteurs face à de nouveaux défis. Actes du colloque, Garoua, Cameroun, 27-31 mai 2002. Montpellier: CIRAD.

Guibert H, Ngamié I, Clavier H, Havard M. 2015. Emerging farms in Northern Cameroon : an economic and social change towards high agricultural productivity. In : Gritti ES, Wery J (ed), Proceedings of the 5th international symposium for farming systems design : FSD5. Montpellier (France): European Society for Agronomy.

Havard M, Enam J, Abakar O. 2000. Les exploitations agricoles des terroirs de référence du Prasac au Cameroun. Résultats de l'enquête exhaustive réalisée entre mars et mai 2000. Garoua, Cameroun: $\mathrm{Irad} /$ Prasac, $26 \mathrm{p}$.

Havard M, Abakar O. 2001. Bilan campagne agricole 2000/2001 dans les terroirs PRASAC, Nord-Cameroun. Garoua, Cameroun: Irad/ Prasac, $34 \mathrm{p}$.
Mathieu B, Marnotte P. 2000. L'enherbement des sols à muskuwaari au Nord-Cameroun. In : $11^{e}$ Coll. Int. sur la biologie des mauvaises herbes. Dijon. 6-8 septembre 2000. Paris: INRA, AFPP (AFPP Annales).

M'Biandoun M, Guibert H, Olina J-P. 2006. Caractérisation de la fertilité du sol en fonction des mauvaises herbes présentes. Tropicultura 24(4): 247-252.

Menyonga, JM, Bezuneh T, Yayock JY, Soumana I. 1994. Progress in food grain research and production in semi-arid Africa. In : Proceedings of the SAFGRAD Inter-Network Conference, Niamey, Niger, 7-14 march 1991. Ouagadougou (Burkina Faso): OAU/ STRC-SAFGRAD.

Minader. 2012. Ministère de l'Agriculture et du Développement Rural-Direction des Enquêtes et des Statistiques Agricoles. AgriStat $n^{\circ}$ 17. Disponible sur http://www.minader.cm/uploads/File/ AGRISTAT\%2017.pdf

Minot N. 2014. Food price volatility in Sub-saharan Africa: has it really increased? Food Policy 45: 45-56. doi: http://dx.doi.org/ 10.1016/j.foodpol.2013.12.008

Naudin K, Gozé E, Balarabe O, Giller KE, Scopel E. 2010. Impact of no tillage and mulching practices on cotton production in North Cameroon: a multi-locational on-farm assessment. Soil \& Tillage Research 108: 68-76. doi: http://dx.doi.org/10.1016/j. still.2010.03.002

Nzossié FEJ, Ndamè JP, Temple L, Dury S, Ndjouenkeu R, Kamdem SM. 2010. L'émergence du maïs dans la consommation alimentaire des ménages urbains au Nord-Cameroun. Économie Rurale 318-319: 65-79. doi: http://dx.doi.org/10.4000/economie rurale. 2769

OCDE/FAO. 2008. Perspectives agricoles de l'OCDE et de la FAO : 2008-2017. Disponible sur http://www.oecd.org/fr/tad/40716017.pdf.

Sissoko F, Coulibaly D, Cissé O, Dugué P. 2015. Évaluation de l'arrière effet de la culture du coton sur la production céréalière en zone cotonnière du Mali. In : Fok M, Ndoye $\mathrm{O}$, Koné S (ed), L'agriculture face aux défis de l'alimentation et de la nutrition en Afrique : quels apports de la recherche dans les pays cotonniers? Gembloux (Belgique): AGRAR-2013. Disponible sur http://www. pressesagro.be/catalogue/reference/132.html

Smith CW. 1995. Crop production, evolution, history and technology. New York: John Wiley \& Sons.

UN-OCHA. 2016. The United Nations - Office for the Coordination of Humanitarian Affairs. Aperçu des besoins humanitaires : Cameroun. Disponible sur https://www.humanitarianresponseinfo/ en/operations/cameroon.

Citation de l'article : Guibert H, Kenne Kueteyim P, Olina Bassala J-P, M'Biandoun M. 2016. Intensifier la culture du maïs pour améliorer la sécurité alimentaire : le producteur du Nord Cameroun y a-t-il intérêt ? Cah. Agric. 25: 65006. 